\title{
MS37-01 | NeW Sensitivity-Enhanced NMR Crystallography Approaches to Investigate Crystallization and Polymorphism in Organic Materials
}

Mollica, Giulia (CNRS, Marseille, FRA)

Polymorphism affects almost $50 \%$ of all the organic compounds referenced in the Cambridge Structural Database. It can have huge economic and practical consequences for industrial applications in pharmacy and energy because different polymorphs display different physicochemical properties. If, on the one hand, it offers great opportunities for tuning the performance of the organic material, on the other hand, manufacture or storageinduced, unexpected, polymorph transitions can compromise the end-use of the solid product. These transformations often imply the formation of metastable forms, which are receiving growing attention because they can offer new crystal forms with improved properties. Today, detection and accurate structural analysis of these - generally transient - forms remain challenging, essentially because of the present limitations in temporal and spatial resolution of the analysis, which prevents rationalization (and hence control) of crystallization processes.

We develop dynamic nuclear polarization (DNP) solid-state NMR approaches to overcome these limitations. In this contribution, I will present some of our latest results showing that cryogenic MAS NMR [1] combined with the sensitivity enhancement provided by DNP [2] can be an efficient way of monitoring the structural evolution of crystallizing solutions with atomic-scale resolution on a time scale of a few minutes.

This project has received funding from the European Research Council (ERC) under the European Union's Horizon 2020 research and innovation programme (grant agreement No 758498).

[1] P. Cerreia-Vioglio et al. Angew. Chem. Int. Ed. 2018, 57, 6619.

[2] P. Cerreia-Vioglio, et al. J. Phys. Chem. Lett. 2019, 10, 1505. 